SLAC-PUB-10091

\title{
Comments on the Fate of unstable orbifolds
}

\author{
Sang-Jin Sin \\ Stanford Linear Accelerator Center, Stanford University, Stanford CA $94305^{*}$ \\ and \\ Department of Physics, Hanyang University, Seoul, 133-791, Korea ${ }^{\dagger}$ \\ June 18, 2018
}

\begin{abstract}
We study the localized tachyon condensation in their mirror Landau-Ginzburg picture. We completely determine the decay mode of an unstable orbifold $C^{r} / Z_{n}, r=1,2,3$ under the condensation of a tachyon with definite R-charge and mass by extending the Vafa's work hep-th/0111105. Here, we give a simple method that works uniformly for all $C^{r} / Z_{n}$. For $C^{2} / Z_{n}$, where method of toric geometry works, we give a proof of equivalence of our method with toric one. For $C^{r} / Z_{n}$ cases, the orbifolds decay into sum of $r$ far separated orbifolds.
\end{abstract}

${ }^{*}$ Work supported partially by the department of Energy under contract number DE-AC03-76SF005515.

${ }^{\dagger}$ Permanent address 


\section{Introduction}

The study of open string tachyon condensation[1] has led to many interesting consequences including classification of the D-brane charge by K-theory. While the closed string tachyon condensation involve the change of the background spacetime and much more difficult, if we consider the case where tachyons can be localized at the singularity, one may expect the maximal analogy with the open string case. Along this direction, the study of localized tachyon condensation was considered in 2] using the brane probe and renormalization group flow and by many others $3,4,4,6,17,8$. The basic picture is that tachyon condensation induces cascade of decays of the orbifolds to less singular ones until the spacetime supersymmetry is restored. Therefore the localized tachyon condensation has geometric description as the resolution of the spacetime singularities.

Soon after, Vafa [3] considered the problem in the Landau-Ginzburg (LG) formulation using the Mirror symmetry and confirmed the result of [2]. In [4], the same problem is studied by using the RG flow as deformation of chiral ring and in term of toric geometry. In [3], Vafa showed that, as a consequence of the tachyon condensation, the final point of the process is sum of two orbifold theories which are far from each other but smoothly connected: one located at north and the other at the south poles of blown up $P^{2}$ singularity of the orbifold in the limit where the radius of the sphere is infinite. Schematically, we can represent this transition by

$$
\mathbb{C}^{2} / \mathbb{Z}_{n\left(k_{1}, k_{2}\right)} \rightarrow \mathbb{C}^{2} / \mathbb{Z}_{p_{1}(*, *)} \oplus \mathbb{C}^{2} / \mathbb{Z}_{p_{2}(*, *)}
$$

with yet unknown generators for the daughter theories.

The purpose of this paper is to determine the the decay mode of unstable orbifolds by working out the generators of orbifold action in daughter theories for $C^{r} / Z_{n} r=1,2,3$. For $\mathbb{C}^{1} / \mathbb{Z}_{n}$, the transition modes are described in earlier works $\left[2, \underline{3}, 4\right.$. For $\mathbb{C}^{2} / \mathbb{Z}_{n\left(k_{1}, k_{2}\right)}$ case, some examples are worked out in 4] using toric geometry and prescription in terms of continued fraction is given. In principle, it can be worked out once numbers are given explicitly. However, that method does not work for $C^{3} / Z_{n}$. Here, we give a simple method that works easily and uniformly for all $C^{r} / Z_{n}$. For $C^{2} / Z_{n}$, we give a proof of equivalence of our method with toric one. To do this we will need to know how the spectrums of chiral primaries are transformed under the condensation of a specific tachyon. 


\section{Mirror symmetry and Orbifolds}

We begin by a summary of Vafa's work [3] on localized tachyon condensation. The orbifold $\mathbb{C}^{r} / Z_{n}$ is defined by the $\mathbb{Z}_{n}$ action given by equivalence relation

$$
\left(X_{1}, \ldots, X_{r}\right) \sim\left(\omega^{k_{1}} X_{1}, \ldots, \omega^{k_{r}} X_{r}\right), \quad \omega=e^{2 \pi i / n} .
$$

We call $\left(k_{1}, \cdots, k_{r}\right)$ as the generator of the $\mathbb{Z}_{n}$ action. The orbifold can be imbedded into the gauged linear sigma model(GLSM) 9]. The vacuum manifold of the latter is described by the D-term constraints

$$
-n\left|X_{0}\right|^{2}+\sum_{i} k_{i}\left|X_{i}\right|^{2}=t
$$

Its $t \rightarrow-\infty$ limit corresponds to the orbifold and the $t \rightarrow \infty$ limit is the $O(-n)$ bundle over the weighted projected space $W P_{k_{1}, \ldots, k_{r}}$. $X_{0}$ direction corresponds to the non-compact fiber of this bundle and $t$ plays role of size of the $W P_{k_{1}, \ldots, k_{r}}$.

By dualizing this GLSM, we get a LG model with a superpotential[10]

$$
W=\sum_{i=0}^{r} \exp \left(-Y_{i}\right)
$$

where twisted chiral fields $Y_{i}$ are periodic $Y_{i} \sim Y_{i}+2 \pi i$ and related to $X_{i}$ by $\operatorname{Re}\left[Y_{i}\right]=\left|X_{i}\right|^{2}$. Introducing the variable $u_{i}:=e^{-Y_{i} / n}$, the D-term constraint is expressed as $e^{-Y_{0}}=e^{t / n} \prod_{i} u^{k_{i}}$. The periodicity of $Y_{i}$ imposes the identification : $u_{i} \sim e^{2 \pi i / n} u_{i}$ which necessitate modding out each $u_{i}$ by $\mathbb{Z}_{n}$. The result is usually described by

$$
\left[W=\sum_{i=1}^{r} u_{i}^{n}+e^{t / n} \prod_{i} u^{k_{i}}\right] / /\left(\mathbb{Z}_{n}\right)^{r-1} .
$$

which describe the mirror Landau-Ginzburg model of the linear sigma model. As a $t \rightarrow-\infty$ limit, mirror of the orbifold is

$$
\left[W=\sum_{i=1}^{r} u_{i}^{n}\right] / /\left(\mathbb{Z}_{n}\right)^{r-1} .
$$

Since it is not ordinary Landau-Ginzburg theory but an orbifolded version, the chiral ring structure of the theory is very different from that of LG model. For example, the dimension of the local ring of the super potential is always $n-1$, regardless of $r$.

We list some properties of orbifolded LG theory for later use. 
The true variable of the theory are $Y_{i}$ not $u_{i}$ related by $u_{i}=e^{-Y_{i} / n}$. As a consequence, monomial basis of the chiral ring is given by

$$
\left\{u_{1}^{p_{1}} u_{2}^{p_{2}} \mid\left(p_{1}, p_{2}\right)=\left(n\left\{j k_{1} / n\right\}, n\left\{j k_{2} / n\right\}\right), j=1, \ldots, n-1\right\},
$$

and $u_{1}^{p_{1}} u_{2}^{p_{2}}$ has weight $\left(p_{1}, p_{2}\right)$ and charge $\left(p_{1} / n, p_{2} / n\right)$.

\section{Fate of the spectrum}

For $\mathbb{C}^{2} / \mathbb{Z}_{n\left(k_{1}, k_{2}\right)}$ case, if one consider the condensation of tachyon in the $l$-th twisted sector that corresponds to chiral ring element $u_{1}^{p_{1}} u_{2}^{p_{2}}$, with $p_{1}=n\left\{l k_{1} / n\right\}$ and $p_{2}=n\left\{l k_{2} / n\right\}$, the theory is given by the super potential

$$
\left[W=u_{1}^{n}+u_{2}^{n}+e^{t / n} u_{1}^{p_{1}} u_{2}^{p_{2}}\right] / / Z_{n}
$$

Consider $u_{2} \sim 0$ and $u_{2}^{n} \sim e^{t / n} u_{1}^{p_{1}} u_{2}^{p_{2}}$ region, which should be described by

$$
\left[W \sim u_{1}^{n}+e^{t / n} u_{1}^{p_{1}} u_{2}^{p_{2}}\right] / / Z_{n}
$$

By introducing the new variables $v_{1}=u_{1}^{n / p_{2}}$ and $v_{2}=e^{t / n p_{2}} u_{1}^{p_{1} / p_{2}} u_{2}$. The single valuedness of

$v_{i}$ induces the $Z_{n}$ but single valuedness of $u_{1}^{n}$ and $u_{1}^{p_{1}} u_{2}^{p_{2}}$ implies that $v_{1}, v_{2}$ are orbifolded by $Z_{p_{2}}$. By substitution, we can express $u_{1}^{q_{1}} u_{2}^{q_{2}}$ in terms of $v_{1}, v_{2}$ :

$$
u_{1}^{q_{1}} u_{2}^{q_{2}}=v_{1}^{Q_{1}} v_{2}^{Q_{2}}
$$

where

$$
\left(Q_{1}, Q_{2}\right)=\left(-p \times q / n, q_{2}\right)
$$

with $p \times q=\left(p_{1} q_{2}-p_{2} q_{1}\right)$. Notice that map $T_{p}^{-}:\left(q_{1}, q_{2}\right) \mapsto\left(Q_{1}, Q_{2}\right)$ is linear map acting on the integrally normalized weight space and can be described by a matrix

$$
T_{p}^{-}=\left(\begin{array}{cc}
p_{2} / n & -p_{1} / n \\
0 & 1
\end{array}\right)
$$

It is working near $u_{2} \sim 0$. It maps $(n, 0) \rightarrow\left(p_{2}, 0\right)$ and $\left(p_{1}, p_{2}\right) \rightarrow\left(0, p_{2}\right)$, or equivalently, $u_{1}^{n} \rightarrow v_{1}^{p_{2}}$ and $u_{1}^{p_{1}} u_{2}^{p_{2}} \rightarrow v_{2}^{p_{2}}$. 
One should notice that $Q_{1}, Q_{2}$ are not integers in general. However, when both $p$ and $q$ are weight vectors of elements of orbifold chiral ring, generated by $\left(k_{1}, k_{2}\right)$, they are integers. This is because if $p=\left(n\left\{l k_{1} / n\right\}, n\left\{l k_{2} / n\right\}\right), q=\left(n\left\{j k_{1} / n\right\}, n\left\{j k_{1} / n\right\}\right), s:=p \times q / n$, then

$$
s=n\left\{l k_{1} / n\right\}\left\{j k_{2} / n\right\}-n\left\{l k_{2} / n\right\}\left\{j k_{1} / n\right\} \in \mathbb{Z}
$$

for any integers $n, k, l, j$. For $k_{1}=1, s=-l\left[j k_{2} / n\right]+j\left[l k_{2} / n\right]$. Especially interesting case will be $q=k=\left(1, k_{2}\right)$, in which case, we have $s=\left[l k_{2} / n\right]=\left(l k_{2}-p_{2}\right) / n$. Geometrically, $s$ is proportional to the area spanned by two vectors $p$ and $q$. Therefore it is zero if $p$ and $q$ are parallel.

The R-charges are determined by the marginality condition. In the original theory, $u_{i}$ has R-charge $1 / n$ since $u_{i}^{n}$ has R-charge 1 . We express this as $R\left[u_{i}^{n}\right]=1$. Therefore $R\left[u_{1}^{p_{1}} u_{2}^{p_{2}}\right]=$ $\left(p_{1}+p_{2}\right) / n$. charge space is defined by the weight space scaled by $1 / n$. So we use the same figure 1 to describe it. The diagonal in charge space is the line connecting $\mathrm{A}(1,0)$ and $\mathrm{B}(0,1)$. Any operator whose $\mathrm{R}$ charge is on this diagonal corresponds to the marginal operator. The points below the diagonal correspond to the relevant operators and tachyonic and those above it correspond to the irrelevant operators. When a tachyon, $\mathrm{P}$, is fully condensed, the marginal line is changed from diagonal line $\mathrm{AB}$ to line $\mathrm{AP}$ or BP. AP gives down-theory and $\mathrm{BP}$ gives the up-theory. $\Delta_{+}$is the cone spanned by $\overrightarrow{O B}$ and $\overrightarrow{O P}$, and similarly $\Delta_{-}$is the cone spanned by $\overrightarrow{O A}$ and $\overrightarrow{O P}$.

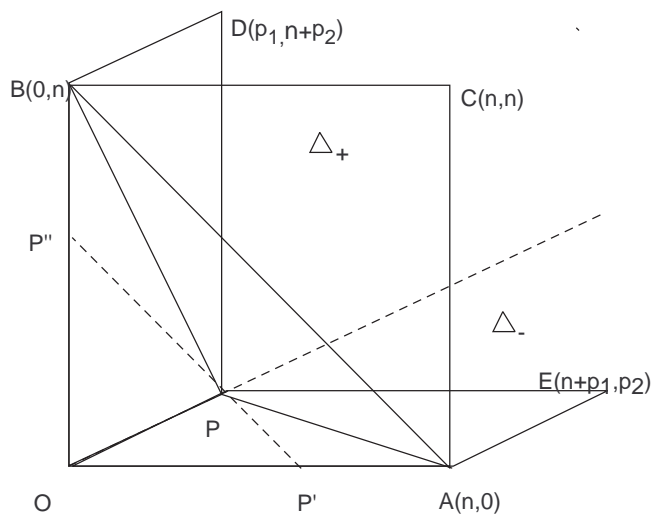

Figure 1: Integrally normalized weight/charge space for $\mathbb{C}^{2} / \mathbb{Z}_{n}$. It can be considered as the space of power of local ring elements. It is defined as a two dimensional torus with size $\mathrm{n} . u_{1}^{n}$ and $u_{2}^{n}$ is located at $\mathrm{A}(\mathrm{n}, 0)$ and $\mathrm{B}(0, \mathrm{n})$ respectively. Under the condensation of tachyon $\mathrm{P}$, the parallelogram $O B D P$ is mapped to the up-theory and $O P E A$ is mapped to the down-theory. Translation parallel to OP is mapped to horizontal in up theory and vertical in down theory.

Let $\mathrm{P}$ be the point $\left(p_{1} / n, p_{2} / n\right)$ in charge space that corresponds to a chiral primary that is 
undergoing condensation, and $\mathrm{Q}$ be any charge point $\left(q_{1} / n, q_{2} / n\right)$ and $\mathrm{A}$, B now corresponds to $(1,0)$ and $(0,1)$. One can work out the action of $T_{p}^{-}$from other point of view. If $\mathrm{P}$ represent the chiral primary of $l$-th twisted sector, $\left(p_{1} / n, p_{2} / n\right):=\left(\left\{l k_{1} / n\right\},\left\{l k_{1} / n\right\}\right)$. Near $u_{2} \sim 0$ region, the marginality condition is changed to $R\left[u_{1}^{p_{1}} u_{2}^{p_{2}}\right]=1, R\left[u_{1}^{n}\right]=1$. In terms of new variable $R\left[v_{i}^{p_{2}}\right]=1$. The linear transformation

$$
\tilde{T}_{p}^{-}:\left(q_{1} / n, q_{2} / n\right) \rightarrow\left(Q_{1} / p_{2}, Q_{2} / p_{2}\right)
$$

can be determined by its action on $\mathrm{P}$ and $(1,0)$. Once $\tilde{T}_{p}^{-}$is decided, we get $T_{p}^{-}$from the relation, $\tilde{T}_{p}^{-}=\frac{n}{p_{2}} T_{p}^{-}$. The result of course agrees with the one given by eq.(3.5). Under this mapping, the lower triangle $\triangle P O A$ in figure 1 in charge space is mapped to the entire $\triangle B O A$, which defines one of theory in the final stage of the tachyon condensation. We call it down-theory. ${ }^{1}$

Similarly, by considering $u_{1} \sim 0$ region, we get the mapping $\tilde{T}_{p}^{+}$that maps the upper triangle $\triangle B O P$ to $\triangle B O A$. By the relation $T_{p}^{+}=\left(p_{1} / n\right) \tilde{T}_{p}^{+}$we can obtain the mapping in weight space:

$$
T_{p}^{+} q=\left(\begin{array}{cc}
1 & 0 \\
-p_{2} / n & p_{1} / n
\end{array}\right)\left(\begin{array}{l}
q_{1} \\
q_{2}
\end{array}\right)=\left(\begin{array}{c}
q_{1} \\
p \times q / n
\end{array}\right)
$$

Notice that $T_{p}^{+}$leaves all the vertical lines in weight space fixed while $T_{p}^{-}$leaves horizontal lines invariant.

Now we ask: given an operator with $q=\left(q_{1}, q_{2}\right)$, should we map with $T_{p}^{+}$or $T_{p}^{-}$? The answer is that we should use the map that gives smaller R-charge. The difference of the R-charge after the mapping is given by

$$
\begin{aligned}
\delta:=R\left[T_{p}^{+} q\right]-R\left[T_{p}^{-} q\right]=\frac{p \times q}{n p_{1} p_{2}}\left(p_{1}+p_{2}-n\right) & <0 \quad \text { if } q \in \Delta_{+} \\
& >0 \quad \text { if } q \in \Delta_{-}
\end{aligned}
$$

where $\Delta_{+}$is the cone spanned by $\overrightarrow{O B}$ and $\overrightarrow{O P}$, and similarly $\Delta_{-}$is the cone spanned by $\overrightarrow{O A}$ and $\overrightarrow{O P}$. Notice that we are condensing relevant operator $p$ so that $p_{1}+p_{2}<n$. The line $B P$ is mapped to the marginal line of a final theory, the up-theory, and the line $A P$ is mapped to that of down-theory. Therefore the emerging picture is following: The parallelogram $O B D P$

\footnotetext{
${ }^{1}$ Conversely, if we require that $\tilde{T}_{p}^{-}$maps $\triangle P O A$ to $\triangle B O A, \tilde{T}_{p}^{-}$is completely determined. The mapping $T^{-}$ in the integrally normalized weight space is induced by $T^{-}=\left(p_{2} / n\right) \tilde{T}^{-}$. The normalization is dictated from the condition that $T$ maps from integer vectors to integer vectors. Finally $T_{p}^{-}(n, 0)=\left(p_{2}, 0\right)$ and $T_{p}^{-}\left(p_{1}, p_{2}\right)=\left(0, p_{2}\right)$ so that the identification $u_{1}^{n}=v^{p_{2}}, u_{1}^{p_{1}} u_{2}^{p_{2}}=v^{p_{2}}$ is dictated.
} 
spanned by $\overrightarrow{O B}$ and $\overrightarrow{O P}$ is mapped to the up-theory whose weight space size is $p_{1}$. Similarly, the parallelogram $O P E A$ spanned by $\overrightarrow{O P}$ and $\overrightarrow{O A}$ is mapped to the down-theory whose weight space size is $p_{2}$. See figure 2. From eq. (3.6), it is easy to see that chiral ring elements of Mother theory are mapped to chiral ring elements of the daughter theories, under the condensation of a chiral ring element. Any operator $q^{\prime}$ outside these two parallelograms can be parallel translated to inside one of above two parallelograms by the vector $\overrightarrow{O P}$ a few times if necessary. In daughter theories, if $q^{\prime} \in \Delta_{+}$, then $T_{p}^{+} q^{\prime}$ can be translated horizontally by $p_{1}$ a few times to a point in the up-theory. Similarly, if $q^{\prime} \in \Delta_{-}$, then $T_{p}^{-} q^{\prime}$ can be translated vertically by $p_{1}$ a few times to a point in the up-theory.

\section{Fate of unstable orbifolds}

\section{1 $\quad \mathbb{C}^{2} / \mathbb{Z}_{n}$}

We now can answer to our main question: what are the generators of final theories? We noticed that there are two theories in the final stage. These two theories are described by the difference of the marginal lines in the weight space: extension of $B P$ or that of $A P$. We call the former as the up-theory, describing $u_{1} \sim 0$ region, and the latter as down-theory, describing the $u_{2} \sim 0$ region. In terms of the charge space, up-theory is obtained by mapping $\tilde{T}_{p}^{+}: \triangle B O P \mapsto \triangle B O A$ and down-theory is obtained by mapping $\tilde{T}_{p}^{-}: \triangle B O P \mapsto \triangle B O A$.

The up-theory is a orbifold $\mathbb{C}^{2} / \mathbb{Z}_{p_{1}}$ and the down theory is another orbifold $\mathbb{C}^{2} / \mathbb{Z}_{p_{2}}$. Let $k=\left(k_{1}, k_{2}\right)$ be the generator of the original theory. Then the generator of the up-theory is given by $T_{p}^{+}(k)=\left(k_{1}, p \times k / n\right)$ and that of the $T_{p}^{-}(k)=\left(-p \times k / n, k_{2}\right)$. Since $\left(k_{1}, k_{2}\right) \sim\left(-k_{1},-k_{2}\right)$ as a generator, one can also use $T_{p}^{-}(-k)=\left(p \times k / n,-k_{2}\right)$ instead of $T_{p}^{-}(k)$. Therefore we can describe the process of condensation of tachyon with charge $p=\left(p_{1}, p_{2}\right)$ as follows:

$$
\mathbb{C}^{2} / \mathbb{Z}_{n\left(k_{1}, k_{2}\right)} \longrightarrow \mathbb{C}^{2} / \mathbb{Z}_{p_{1}\left(k_{1}, p \times k / n\right)} \oplus \mathbb{C}^{2} / \mathbb{Z}_{p_{2}\left(-p \times k / n, k_{2}\right)}
$$

To simplify the notation, we use $n\left(k_{1}, k_{2}\right)$ for $\mathbb{C}^{2} / \mathbb{Z}_{k_{1}, k_{2}}$ and $s=p \times k / n$. Then,

$$
n\left(k_{1}, k_{2}\right) \underset{\left(p_{1}, p_{2}\right)}{\longrightarrow} p_{1}\left(k_{1}, s\right) \oplus p_{2}\left(-s, k_{2}\right) .
$$

Especially interesting cases are those when one of $k_{i}$ is 1 .

$$
n(1, k) \underset{\left(p_{1}, p_{2}\right)}{\longrightarrow} p_{1}(1, s) \oplus p_{2}(-s, k) \text {, if } k_{1}=1, k_{2}=k .
$$


In order to check the validity of our method, we check that all of examples studied in APS and HKMM, where some of $k_{1}=1$ case is considered.

1. $2 l(1,-1) \overrightarrow{(l, l)} l(1,-1) \oplus l(1,-1)$, with $s=-1$. APS example 5.2

2. $2 l(1,3) \underset{(l, l)}{\longrightarrow} l(1,1) \oplus l(1,-3)$, with $s=1$. APS Ex.5.3

3. $5(1,3) \underset{(2,1)}{\longrightarrow} 2(1,1) \oplus \mathbb{C}^{2}$, with $s=1$. A generic tachyon condensation. APS Ex.5.4

4. $n(1,1) \underset{(p, p)}{\longrightarrow} p(1,0) \oplus p(0,1)$ : all charges are on the diagonal $q_{1}=q_{2}$ line, so $s=0$. This is two copies of $\mathbb{C}^{1} / \mathbb{Z}_{p} \times \mathbb{C}$.

5. $n(1,-1) \underset{(l, n-l)}{\longrightarrow} l(1,-1) \oplus^{\prime} n-l^{\prime}(1,-1)$ : all charges are on the marginal line $q_{1}+q_{2}=n$. $s=-1$.

6. $n(1,-3) \underset{(j,-3 j)}{\longrightarrow} j(1,-\alpha) \oplus \alpha^{\prime} n-3 j^{\prime}(\alpha,-3)$, where $\alpha=[3 j / n]+1$. Notice $p=(j,-3 j) \equiv$ $(j, \alpha n-3 j)$, so that $s=-\alpha . \alpha=1$ case is the example 4.3.3 of HKMM.

Now, what about the generic case where neither $k_{1}$ nor $k_{2}$ is equal to 1 ? We first discuss the non-reducible cases where $\left\{l k_{i} / n\right\} \neq 0$ for any $l=1, \ldots, n-1$. This is the case if $k_{i}$ and $n$ are relatively prime. Then we can choose a new generator $(1, k)$ such that

$$
\{j(1, k) \mid j=1, \ldots, n-1\}=\left\{l\left(k_{1}, k_{2}\right) \mid l=1, \ldots, n-1\right\},
$$

because we can find $k$ such that for any given $l, l k_{1}=j \bmod n$ and $l k_{2}=j k \bmod n$ for some $j$. In fact $k$ is given by

$$
k \equiv k_{2} / k_{1} \bmod n .
$$

Therefore generic case is isomorphic to $n(1, k)$ type. $^{2}$ For example, $11(2,3)$ is identical to $11(1,7)$ and also to $11(8,1)$, since $3 / 2 \equiv 7,2 / 3 \equiv 8 \bmod 11$.

Sometimes we meet situation where $s=0$, where we need more care. For example, if we condensate the generator $(1, k)$ itself, eq. (4.3) predict that

$$
n(1, k) \rightarrow 1(1,0) \oplus k(0, k) .
$$

For the first element $1(1,0)$, it is correct since the upper triangle does not contain any tachyon operator. However, for the second element, this can not be true since we have non-trivial

\footnotetext{
${ }^{2}$ So far we proved this fact in the conformal filed theory level before GSO projection.
} 

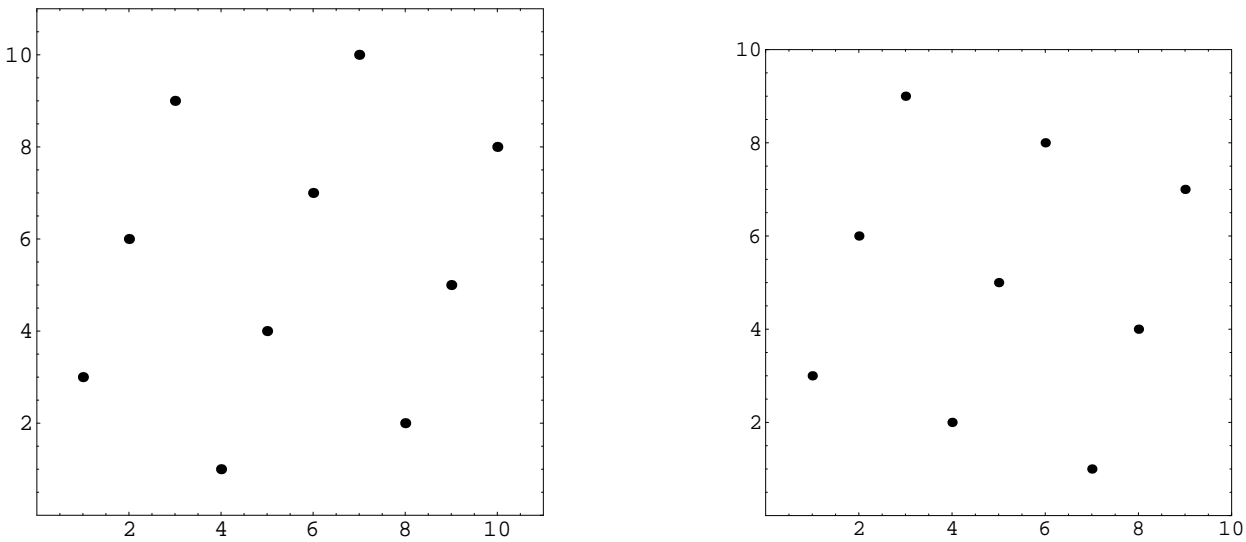

Figure 2: Charges of 11(1,3) (left) and 10(1,3) (right) in Weight space.

operator in the lower triangle. This is clear from 11(1,3) model described in the figure 2 , where all twisted tachyons coming from chiral primaries are given in the figure 2, $s=0$ is caused by the fact that $p$ and $(1, k)$ are parallel. So we need to choose a generator of the lower triangle other than $(1, k)$. Assuming $k$ and $n$ are relatively prime, $k$ has multiplicative inverse modulo $n$, which we denote by $k^{-1}$. We also introduce $s^{\prime}=p \times\left(k^{-1}, 1\right) / n$. Then we have $n(1, k)=n\left(k^{-1}, 1\right)$. Now the image of the new generator under $T_{p}^{-}$is $\left(-s^{\prime}, 1\right)$. It is easy to show that $k s^{\prime}=s-a p_{2}$ where $a$ is defined by $k^{-1} k=n a+1$. Therefore $p_{2}(-s, k)=p_{2}\left(-s^{\prime}, 1\right)$ if $s$ is not 0 . So we get

$$
n(1, k) \underset{\left(p_{1}, p_{2}\right)}{\longrightarrow} p_{1}(1, s) \oplus p_{2}\left(-s^{\prime}, 1\right) .
$$

The equations (4.3), (4.7) are the main formula of this section. When one of $s, s^{\prime}$ is 0 and the other is not, we should use the non-zero one. For example, when the condensing operator is of the form $j\left(k^{-1}, 1\right), s^{\prime}=0$ and it is better to use $p_{2}(-s, k)$ for the exactly same reason as we use $p_{2}\left(-s^{\prime}, 1\right)$ when $s=0$. When $s s^{\prime} \neq 0$ two are equivalent in conformal field theory level. ${ }^{3}$ We give a few examples below. If we condensate an operator with $p=j(1, k)$, its band number $G:=[j / n]+[j k / n]=0$ and $s=0$. However, $s^{\prime}=j(1, k) \wedge\left(k^{-1}, 1\right)=-a j \neq 0$ unless $k=1($ or, $a=0)$. The transition is described as

$$
n(1, k) \underset{j(1, k)}{\longrightarrow} j(1,0) \oplus j k(j a, 1) .
$$

More explicitly, for $p=(2,6)$ in $11(1,3), j=2, s=0, k=3, k^{-1}=4,4 \cdot 3=11 \cdot 1+1$ hence

\footnotetext{
${ }^{3}$ For string theory level, two prescriptions are different if $s$ and $s^{\prime}$ does not have the same G-parity (even or odd-ness). we need to use the one that has the same parity as that of $\mathrm{k}$. This will be discussed further in later section.
} 


\begin{tabular}{|c|c|c|c|c|}
\hline$j$ & $\left(p_{1}, p_{2}\right)$ & $G=[3 j / 11]$ & $n-\left(p_{1}+p_{2}\right)$ & process \\
\hline \hline 1 & $(1,3)$ & 0 & 7 & $11(1,3) \mapsto 1(1,0) \oplus 3(1,1)$ \\
\hline 2 & $(2,6)$ & 0 & 3 & $11(1,3) \mapsto 2(1,0) \oplus 6(2,1)$ \\
\hline 3 & $(3,9)$ & 0 & -1 & irrelevant process \\
\hline 4 & $(4,1)$ & 1 & 6 & $11(1,3) \mapsto 4(1,1) \oplus 1(0,1)$ \\
\hline 5 & $(5,4)$ & 1 & 2 & $11(1,3) \mapsto 5(1,1) \oplus 4(1,1)$ \\
\hline 6 & $(6,7)$ & 1 & -2 & irrelevant process \\
\hline 7 & $(7,10)$ & 1 & -6 & irrelevant process \\
\hline 8 & $(8,2)$ & 2 & 1 & $11(1,3) \mapsto 8(1,2) \oplus 2(0,1)$ \\
\hline 9 & $(9,5)$ & 2 & -3 & irrelevant process \\
\hline 10 & $(10,8)$ & 2 & -7 & irrelevant process \\
\hline
\end{tabular}

Table 1: All possible tachyon condensation process in 11(1,3) model. We should consider only the processes given by relevant operators, namely those with $n-\left(p_{1}+p_{2}\right)>0$, otherwise it is a process by an irrelevant operator which disappears in the infrared limit.

$a=1$ and $s^{\prime}=-2$ so that

$$
11(1,3) \underset{(2,6)}{\longrightarrow} 2(1,0) \oplus 6(2,1) .
$$

Notice that $6(2,1)$ contains an operator $(0,3)$ so that this is a reducible orbifold. Even in the case we start with irreducible orbifold, we can get reducible orbifold as a result of tachyon condensation. This happen if and only if there is an operator sitting on the line which connect $(0,0)$ and the condensing one, $p$. We tabulated all possible tachyon condensation processes for model $11(1,3)$ and 10(1,3) in table 1 and table respectively.

\subsection{Equivalence of LG and Toric method in $\mathbb{C}^{2} / \mathbb{Z}_{n}$}

Here we show the equivalence of our description of tachyon decay in mirror LG model with that in toric geometry [11] for the case of $\mathbb{C}^{2} / \mathbb{Z}_{n}$. We will show that the transition in LG picture

$$
n(1, k) \underset{\left(p_{1}, p_{2}\right)}{\longrightarrow} p_{1}(1, s) \oplus p_{2}\left(-s^{\prime}, 1\right),
$$

with $s=p \wedge(1, k) / n, s^{\prime}=p \wedge\left(k^{-1}, 1\right) / n$ has corresponding description in toric picture

$$
n(k) \underset{\left(n^{\prime},-k^{\prime}\right)}{\longrightarrow} n^{\prime}\left(k^{\prime}\right) \oplus n^{\prime \prime}\left(k^{\prime \prime}\right),
$$




\begin{tabular}{|c|c|c|c|c|}
\hline$j$ & $\left(p_{1}, p_{2}\right)$ & $G=[3 j / 10]$ & $n-\left(p_{1}+p_{2}\right)$ & process \\
\hline \hline 1 & $(1,3)$ & 0 & 6 & $10(1,3) \mapsto 1(1,0) \oplus 3(0,1)$ \\
\hline 2 & $(2,6)$ & 0 & 2 & $10(1,3) \mapsto 2(1,0) \oplus 6(0,1)$ \\
\hline 3 & $(3,9)$ & 0 & -2 & irrelevant process \\
\hline 4 & $(4,2)$ & 1 & 4 & $10(1,3) \mapsto 4(1,1) \oplus 2(1,1)$ \\
\hline 5 & $(5,5)$ & 1 & 0 & $10(1,3) \mapsto 5(1,1) \oplus 5(1,2)$ \\
\hline 6 & $(6,8)$ & 1 & -4 & irrelevant process \\
\hline 7 & $(7,1)$ & 2 & 2 & $10(1,3) \mapsto 7(1,2) \oplus 1(0,1)$ \\
\hline 8 & $(8,4)$ & 2 & -2 & irrelevant process \\
\hline 9 & $(9,7)$ & 2 & -6 & irrelevant process \\
\hline
\end{tabular}

Table 2: All possible localized tachyon condensation in model 10(1,3).

where

$$
n^{\prime \prime}=k n^{\prime}-n k^{\prime} \text { and }-k^{\prime \prime}=c n^{\prime}-d k^{\prime}
$$

with integer $c, d$ satisfying $c n-d k=1 .{ }^{4}$ Notice that it is assumed that $k, n$ is relatively prime.

The data of weight diagram of LG model can be related to that of toric geometry by a linear map $U: L G \rightarrow$ Toric and its inverse $U^{-1}$ :

$$
U=\left(\begin{array}{cc}
1 & 0 \\
-k / n & 1 / n
\end{array}\right), \quad U^{-1}=\left(\begin{array}{cc}
1 & 0 \\
k & n
\end{array}\right)
$$

The weight $\left(p_{1}, p_{2}\right)$ of the condensing tachyon is related to the corresponding toric data $n^{\prime}\left(k^{\prime}\right)$ by

$$
\left(\begin{array}{l}
p_{1} \\
p_{2}
\end{array}\right)=U^{-1}\left(\begin{array}{c}
n^{\prime} \\
-k^{\prime}
\end{array}\right)=\left(\begin{array}{c}
n^{\prime} \\
k n^{\prime}-n k^{\prime}
\end{array}\right)
$$

which gives $p_{1}, p_{2}$ :

$$
p_{1}=n^{\prime}, \quad p_{2}=k n^{\prime}-n k^{\prime}
$$

from which $s$ can calculated in terms of toric data:

$$
s=p \wedge(1, k) / n=\left(n^{\prime}, k n^{\prime}-n k^{\prime}\right) \wedge(1, k) / n=k^{\prime} .
$$

\footnotetext{
${ }^{4}$ If $(c, d)$ is a solution of this equation, $\left(c+k^{\prime} m, d+n^{\prime} m\right)$ is also a solution. The result is the $\left(n^{\prime \prime} .-k^{\prime \prime}\right) \rightarrow$ $\left(n^{\prime \prime},-k^{\prime \prime}+n^{\prime \prime} m\right)$ which is just an $S L_{2} Z$ transformation $\left(\begin{array}{ll}1 & 0 \\ m & 1\end{array}\right)$ which corresponds to a holomorphic coordinate transformation of a toric variety.
} 
Now, since $p_{1}(1, s)$ is trivially equal to $n^{\prime}\left(k^{\prime}\right)$, we only need to show the equivalence of $p_{2}\left(-s^{\prime}, 1\right)$ with $n^{\prime \prime}\left(k^{\prime \prime}\right)$. The question is whether $k^{\prime \prime} \equiv-s^{\prime} \bmod p_{2}$ or equivalently,

$$
\left(c n^{\prime}-d k^{\prime}\right) \equiv\left(p_{1}-k^{-1} p_{2}\right) / n \bmod p_{2}
$$

is true or not. Multiplying both sides by $k,\left(c n^{\prime}-d k^{\prime}\right) k \equiv\left(k p_{1}-k^{-1} k p_{2}\right) / n \bmod p_{2}$. Using $c n-d k=1, s=\left(k p_{1}-p_{2}\right) / n$ and $k^{-1} k=1+a n$, left hand side is equal to $k^{\prime}$ and right hand side is $s-a p_{2}$. From $s=k^{\prime}$, we now have proved eq.(4.17). Now $-k k^{\prime \prime}=k s^{\prime} \bmod p_{2}$ implies $k^{\prime \prime} \equiv-s^{\prime} \bmod p_{2}$, provided $k$ and $p_{2}$ are relatively prime to each other, completing the proof of our desired result.

Remark: It is interesting to observe that for a general chiral ring element $q=(j, n\{j k / n\})$, $U q=(j, k \times q / n)=\tilde{T}_{k}^{+}(q / n)=(j,-[j k / n])$, which means formally, $U$ coincide with tachyon condensation mapping for generator condensation. This fact directly generalizes to the general $\left(k_{1}, k_{2}\right)$.

\section{$4.3 \mathbb{C}^{3} / \mathbb{Z}_{n}$}

We now describe what happens in $\mathbb{C}^{3} / \mathbb{Z}_{n}$ case. Our method is especially useful in the present case since it applies in this case without any difficulty while toric method does not work here [7. When a tachyon with weight vector $\left(p_{1}, p_{2}, p_{3}\right) / n$, the mirror LG is described by the superpotential

$$
\left[W=u_{1}^{n}+u_{2}^{n}+u_{3}^{n}+e^{\frac{t}{n}} u_{1}^{p_{1}} u_{2}^{p_{2}} u_{3}^{p_{3}}\right] / /\left(\mathbb{Z}_{n}\right)^{2} .
$$

By considering $u_{j} \sim 0$ region for $j=1,2,3$, we get the tachyon maps $T_{p}^{(j)}$ 's [12] given by

$$
T_{p}^{(1)}=\left(\begin{array}{ccc}
1 & 0 & 0 \\
-p_{2} / n & p_{1} / n & 0 \\
-p_{3} / n & 0 & p_{1} / n
\end{array}\right), T_{p}^{(2)}=\left(\begin{array}{ccc}
p_{2} / n & -p_{1} / n & 0 \\
0 & 1 & 0 \\
0 & -p_{3} / n & p_{2} / n
\end{array}\right), T_{p}^{(3)}=\left(\begin{array}{ccc}
p_{3} / n & 0 & -p_{1} / n \\
0 & p_{3} / n & -p_{2} / n \\
0 & 0 & 1
\end{array}\right),
$$

which play similar role of $T_{p}^{ \pm}$in $\mathbb{C}^{2} / Z_{n}$. Let $k=\left(k_{1}, k_{2}, k_{3}\right)$ be the generator of mother theory. Then the the generator of the daughter theories are given by $k^{(j)}:=T_{p}^{(j)} k, j=1,2,3$. Namely the orbifold transition rule is given by

$$
n\left(k_{1}, k_{2}, k_{3}\right) \underset{\left(p_{1}, p_{2}, p_{3}\right)}{\longrightarrow} p_{1}\left(k_{1}, s_{12}, s_{13}\right) \oplus p_{2}\left(s_{21}, k_{2}, s_{23}\right) \oplus p_{3}\left(s_{31}, s_{32}, k_{3}\right),
$$

where $s_{j i}=p_{j} k_{i}-p_{i} k_{j}$. Notice that there exists a simple formula

$$
k_{i}^{(j)}=k_{i} \delta_{j i}+s_{j i}
$$

This is one of the main result of this paper. 


\section{Conclusion}

In this paper, we determined the the decay mode of unstable orbifolds by working out the generators of orbifold action in daughter theories for $C^{r} / Z_{n} r=1,2,3$. We gave a simple method that works easily and uniformly for all $C^{r} / Z_{n}$. For $C^{2} / Z_{n}$, we give a proof of equivalence of our method with toric one. Our method trivially reproduced all of known cases worked out by brane probe 2] or toric method [4. For $C^{3} / Z_{n}$ cases, the unstable orbifolds decay into sum of three orbifolds.

Our discussion uses $\mathrm{N}=2$ worldsheet SUSY essentially. It would be very interesting if we can get the the same result without using it.

\section{Acknowledgement}

I would like to thank Allan Adams, Keshav DasGupta and Eva Silverstein for discussions. This work is partially supported by the Korea Research Foundation Grant (KRF-2002-013-D00030) and by KOSEF Grant 1999-2-112-003-5. .

\section{References}

[1] A. Sen, "Non-BPS states and branes in string theory," arXiv:hep-th/9904207, and references citing it; For review, see

A. Sen, "Stable non-BPS states in string theory," JHEP 9806, 007 (1998) arXiv:hep-th/9803194, and references therein.

[2] A. Adams, J. Polchinski and E. Silverstein, "Don't panic! Closed string tachyons in ALE space-times," JHEP 0110, 029 (2001) arXiv:hep-th/0108075.

[3] C. Vafa, "Mirror symmetry and closed string tachyon condensation," arXiv:hep-th/0111051.

[4] J. A. Harvey, D. Kutasov, E. J. Martinec and G. Moore, "Localized tachyons and RG flows," arXiv:hep-th/0111154.

[5] A. Dabholkar and C. Vafa, "tt* geometry and closed string tachyon potential," arXiv:hep-th/0111155. 
[6] A. Dabholkar, "Tachyon condensation and black hole entropy," Phys. Rev. Lett. 88, 091301 (2002) arXiv:hep-th/0111004.

[7] T. Sarkar, "Brane probes, toric geometry, and closed string tachyons," Nucl. Phys. B 648, 497 (2003) arXiv:hep-th/0206109.

[8] Y. Michishita and P. Yi, "D-brane probe and closed string tachyons," Phys. Rev. D 65, 086006 (2002) arXiv:hep-th/0111199,

Soonkeon Nam and Sang-Jin Sin, "Condensation of Localized Tachyons and Spacetime Supersymmetry", arXiv:hep-th/0201132,

Sang-Jin Sin, "Tachyon mass, c-function and Counting localized degrees of freedom," Nucl. Phys. B637 (2002)395, arXiv:hep-th/0202097;

R. Rabadan and J. Simon, "M-theory lift of brane-antibrane systems and localised closed string tachyons," JHEP 0205, 045 (2002) arXiv:hep-th/0203243;

A. M. Uranga, "Localized instabilities at conifolds," arXiv:hep-th/0204079,

T. Suyama, "Closed string tachyons and RG flows," JHEP 0210, 051 (2002) arXiv:hep-th/0210054;

J. L. Barbon and E. Rabinovici, "Remarks on black hole instabilities and closed string tachyons," arXiv:hep-th/0211212;

Y. H. He, "Closed string tachyons, non-supersymmetric orbifolds and generalised McKay correspondence," arXiv:hep-th/0301162,

E. J. Martinec and G. Moore, "On decay of K-theory," arXiv:hep-th/0212059.

[9] E. Witten, "Phases of $\mathrm{N}=2$ theories in two dimensions," Nucl. Phys. B 403, 159 (1993) arXiv:hep-th/9301042.

[10] K. Hori and C. Vafa, "Mirror symmetry," arXiv:hep-th/0002222

[11] E. J. Martinec, "Defects, decay, and dissipated states," arXiv:hep-th/0210231.

[12] Sunggeun Lee and Sang-Jin Sin, "Chiral rings and GSO projection in Orbifolds", arXiv:hep-th/0307xxx. 